\title{
Amperometric Monitoring of Sensory-Evoked Dopamine Release in Awake Larval Zebrafish
}

\author{
Chun-feng Shang, ${ }^{1}$ Xiao-quan Li, ${ }^{1}$ Chen Yin, ${ }^{1,2}$ Bing Liu, ${ }^{4}$ Yu-fan Wang, ${ }^{1,2}$ Zhuan Zhou, ${ }^{4}$ and Jiu-lin $\mathrm{Du}^{1,2,3}$ \\ ${ }^{1}$ Institute of Neuroscience, State Key Laboratory of Neuroscience, CAS Center for Excellence in Brain Science and Intelligence Technology, Shanghai \\ Institutes for Biological Sciences, Chinese Academy of Sciences, Shanghai 200031, China, ${ }^{2}$ Graduate School, University of Chinese Academy of Sciences, \\ Shanghai 200031, China, ${ }^{3}$ School of Life Science and Technology, ShanghaiTech University, Shanghai 200031, China, and ${ }^{4}$ State Key Laboratory of \\ Membrane Biology, Institute of Molecular Medicine and Peking-Tsinghua Center for Life Sciences and PKU-IDG/McGovern Institute for Brain Research, \\ Peking University, Beijing 100871, China
}

Dopamine plays crucial roles in a broad spectrum of brain functions, and neural circuit mechanisms underlying dopaminergic regulation have been intensively studied in the past decade. As larval zebrafish have relatively simple and highly conserved dopaminergic systems, it can serve as an ideal vertebrate animal model to tackle this issue at a whole-brain scale. For this purpose, it is important to develop methods for monitoring endogenous dopamine release in intact larval zebrafish. Here, we developed a real-time method to monitor dopamine release at high spatiotemporal resolution in the brain of awake larval zebrafish using carbon fiber microelectrodes. As an example for application, we combined this method with genetic tools and in vivo calcium imaging and found that food extract can activate pretectal dopaminergic neurons, which in turn release dopamine at the visual center through their projection, providing a dopaminergic circuit mechanism for olfactory modulation of visual functions. Thus, our study demonstrates, for the first time, the utility of carbon fiber microelectrodes for monitoring sensory-evoked dopamine release in the brain of an awake small organism.

Key words: carbon fiber electrode; cross-modal; dopamine; electrochemistry; pretectum; zebrafish

\section{Significance Statement}

With carbon fiber microelectrodes, we have succeeded in monitoring sensory-evoked dopamine release in the brain of an awake small organism for the first time. By elucidating the circuitry origin of the dopamine release, we illustrated the potential application of this method in dissection of the neural circuitry mechanisms underlying dopaminergic neuromodulation.

\section{Introduction}

Dopaminergic modulation plays multifaceted roles in brain functions ranging from sensory processing, sensorimotor trans-

Received Aug. 12, 2015; revised Sept. 19, 2015; accepted Sept. 26, 2015.

Author contributions: C.-f.S. and J.-I.D. designed research; C.-f.S. and Y.-f.W. performed research; X.-q.L., C.Y., B.L., and Z.Z. contributed unpublished reagents/analytic tools; C.-f.S. analyzed data; C.-f.S. and J.-I.D. wrote the paper.

This work was supported by Chinese Academy of Sciences Strategic Priority Research Program Grant XDB02040003, Ministry of Science and Technology of China "973" Program Grants 2011CBA00400 and 2012CB945101, Natural Science Foundation of China National Outstanding Young Scientist Program Grant 31325011 and Young Scientist Program Grant 31400954, and Science and Technology Commission of Shanghai Shanghai Subject Chief Scientist Program Grant 14XD1404100 and Young Scientist Program Grant $13 Z$ Z1464000. We thank Dr. Marc Ekker for providing the DAT promoter construct.

The authors declare no competing financial interests.

Correspondence should be addressed to either of the following: Dr. Jiu-lin Du, Institute of Neuroscience, State Key Laboratory of Neuroscience, CAS Center for Excellence in Brain Science and Intelligence Technology, Shanghai Institutes for Biological Sciences, Chinese Academy of Sciences, 320 Yue-Yang Road, Shanghai 200031, China, E-mail: forestdu@ion.ac.cn; or Dr. Zhuan Zhou, State Key Laboratory of Membrane Biology, Institute of Molecular Medicine and Peking-Tsinghua Center for Life Sciences and PKU-IDG/McGovern Institute for Brain Research, Peking University, Beijing 100871, China, E-mail: zzhou@pku.edu.cn.

DOI:10.1523/JNEUROSCI.3050-15.2015

Copyright $\odot 2015$ the authors $\quad 0270-6474 / 15 / 3515291-04 \$ 15.00 / 0$ formation, motor control, motivation, reinforcement learning to decision-making (Schultz et al., 1997; Wise, 2004; Mu et al., 2012; Marder et al., 2014). Through widely spreading afferents of dopaminergic neurons and various types of dopamine receptors elaborately distributed across different brain areas, dopamine shapes these neural functions in complex and variable ways (Lammel et al., 2011; Marder et al., 2014). Electrochemical recording provides a high spatiotemporal resolution and has demonstrated its power in monitoring dopamine release in the brains of various animals (Phillips et al., 2003; Dommett et al., 2005; Clark et al., 2010; Wang et al., 2011; Howe et al., 2013; Rees et al., 2015). However, the complexity of dopaminergic systems in mammalian brains hinders thorough dissection of neural circuit mechanisms underlying dopaminergic neuromodulation.

The larval zebrafish with a small and transparent brain has become an attractive vertebrate model for system neuroscience research (Friedrich et al., 2010). Because of its simple but highly conserved dopaminergic systems in comparison with mammals and high accessibility for electrophysiology and optophysiology assays, larval zebrafish offer an ideal opportunity for dissecting 
A

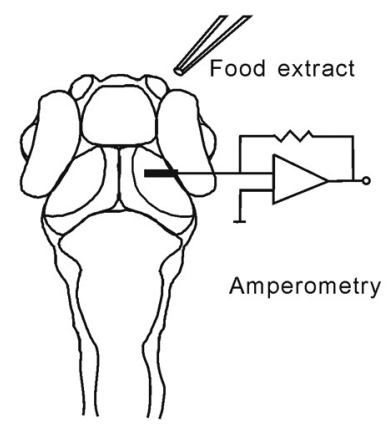

B

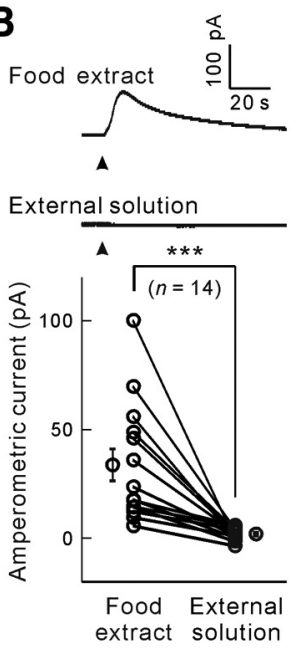

C

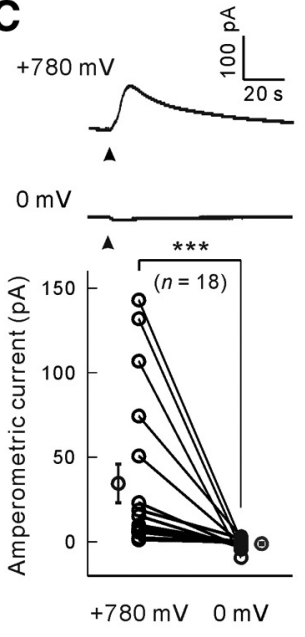

D

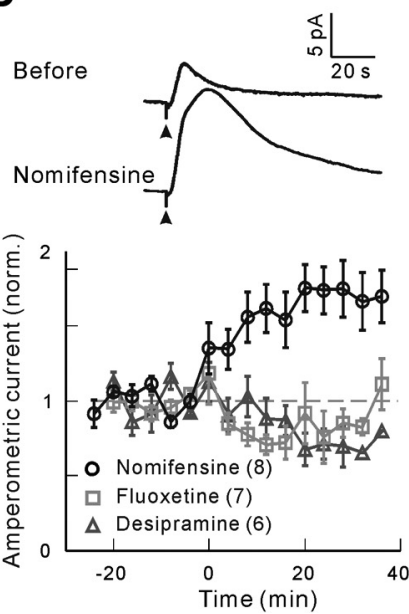

Figure 1. C-fiber microelectrode-based amperometric measurement of food extract-evoked dopamine release at the $0 \mathrm{~T}$ in awake larval zebrafish. $A$, Experimental paradigm. A C-fiber microelectrode was placed in the neuropil of the $0 \mathrm{~T}$, and food extract was applied pneumatically at the ipsilateral nostril through a glass micropipette. $\boldsymbol{B}$, Amperometric current responses evoked by application (arrowhead) of food extract or external solution when the C-fiber microelectrode was held at $780 \mathrm{mV}$. Top, Sample trace showing food extract-evoked response. Middle, Sample trace showing external solution-evoked response. Bottom, Summary. Each symbol represents data obtained from individual larvae, and the data obtained from the same larva are connected by a line. $C$, Amperometric current responses evoked by food extract (arrowhead) when the C-fiber microelectrode was held at 780 or $0 \mathrm{mV}$. Top, Sample trace at $780 \mathrm{mV}$. Middle, Sample trace at 0 $\mathrm{mV}$. Bottom, Summary. Each symbol represents data obtained from individual larvae, and the data obtained from the same larva are connected by a line. $\boldsymbol{D}$, Amperometric current responses evoked by food extract when the larva was treated with the dopamine reuptake blocker nomifensine $(32 \mu \mathrm{m})$, the serotonin reuptake blocker fluoxetine $(0.8 \mu \mathrm{m})$, or the norepinephrine reuptake blocker desipramine $(165 \mu \mathrm{M})$. Top, Sample traces before and after bath application of nomifensine. Bottom, Mean changes with time. The drugs were applied at time $0 .{ }^{* * *} p<0.001$. Error bars indicate SEM.

neural circuitry and synaptic mechanisms underlying neuromodulation (McLean and Fetcho, 2004; Friedrich et al., 2010; Tay et al., 2011; Mu et al., 2012). This would benefit substantially in vivo electrochemical monitoring of dopamine release in the brain of larval zebrafish. Here, using carbon fiber (C-fiber) microelectrodes, we established a real-time electrochemical assay to monitor sensory-evoked dopamine release in awake zebrafish larvae, verifying the utility of C-fiber microelectrodes for in vivo measurement of sensory-evoked dopamine release in small awake organisms for the first time. As an example, using this method, we revealed a novel cross-modal modulation mechanism that olfactory inputs evoke dopamine release at the visual center via a specific cluster of dopaminergic neurons.

\section{Materials and Methods}

Animal husbandry. Adult zebrafish (Danio rerio) were maintained in the National Zebrafish Resources of China (Shanghai, China) at $28^{\circ} \mathrm{C}$ according to a standard protocol (Zhang et al., 2010). Larvae at 6-8 d post-fertilization were used. All the experimental protocols follow the guidelines of Institute of Neuroscience, Shanghai Institute for Biological Sciences, Chinese Academy of Sciences.

In vivo amperometric measurement. Zebrafish larvae were paralyzed with bath-applied $\alpha$-bungarotoxin (100 $\mu \mathrm{g} / \mathrm{ml}$, Tocris Bioscience) for $10-15$ min before embedding in $1 \%-2 \%$ low melting-point agarose (Invitrogen). Agarose around the nose was gently removed with a glass micropipette for odorant delivery. Electrochemistry experiments were performed at room temperature $\left(22^{\circ} \mathrm{C}-25^{\circ} \mathrm{C}\right)$ with an extracellular solution, $\mathrm{pH}$ 7.4, containing the following (in $\mathrm{mM}$ ): $134 \mathrm{NaCl}, 2.9 \mathrm{KCl}, 2.1$ $\mathrm{CaCl}_{2}, 1.2 \mathrm{MgCl}_{2}, 10$ HEPES, and 10 glucose (Sigma-Aldrich). C-fiber microelectrodes were prepared and used to monitor dopamine release as described previously (Huang et al., 2007; Wang et al., 2011; Kang et al., 2014; Xu et al., 2015). Briefly, a C-fiber was preloaded into a glass capillary ( $1 \mathrm{~mm}$ in diameter), which was then pulled by a vertical puller (Narishige PP830) to provide two glass-insulated pre-electrodes. The pre-electrodes were trimmed for parameters meeting our experimental requirements ( $\sim 5 \mu \mathrm{m}$ in diameter and $\sim 30 \mu \mathrm{m}$ in length). The exposed tip was positioned at the anterior neuropil of the optic tectum (OT) through a tiny wound around the midline, $100-200 \mu \mathrm{m}$ posterior to the recording site. Amperometry was performed at a constant holding potential of $780 \mathrm{mV}$ relative to an $\mathrm{Ag} / \mathrm{AgCl}$ reference electrode. Amperometric currents were acquired and low-pass filtered at $1 \mathrm{kHz}$ with a $700 \mathrm{~B}$ Multiclamp patch-clamp amplifier (Molecular Devices) and sampled at $2 \mathrm{kHz}$ with a 1440A Digitizer (Molecular Devices).

Calcium imaging. In vivo calcium imaging of pretectal dopaminergic neurons was performed on $\operatorname{Tg}($ dat:GCaMP6s) larvae with a customized light sheet illumination microscope. Images were taken with a high frame rate sCMOS camera (Hamamatsu ORCA Flash 4.0) at $50 \mathrm{~Hz}$. Calcium signals of the ROI were corrected for drift and normalized to its baseline before measuring the response.

Olfactory stimulation. Food extract was prepared freshly before each experiment. Dry fish food was soaked in system water, and the extract was filtered before using. Filtered food extract was pneumatically delivered with a picrospritzer (Picrospritzer III, Parker Instruments) through a glass micropipette (tip opening: $10-20 \mu \mathrm{m}$ ) positioned $90 \mu \mathrm{m}$ away from the nose of zebrafish larvae. To evoke robust olfactory responses, one trial of food extract delivery consisted of five 10-psi pulses of air puff, each lasting $100 \mathrm{~ms}$ and applied at $1 \mathrm{~Hz}$.

Pharmacology. Nomifensine and desipramine were purchased from Sigma-Aldrich, and fluoxetine was from Tocris Bioscience. To suppress the reuptake of monoamines, stock solutions of corresponding blockers were diluted in the bath solution and resulted in the working concentrations described in the main text.

Laser ablation. Two-photon laser ablation was performed with a FluoView 1000 confocal/two-photon laser-scanning microscope (Olympus). High-power two-photon laser (850 nm, Chameleon Vision, Coherent) was focused on green fluorescent protein (GFP)-expressing dopaminergic neurons and scanned along a coiled track filling the territory of the cell body. The scanning was stopped once the targeted cell turned gray. A small bubble was formed occasionally. Ablation of all pretectal dopaminergic neurons in one larva usually took several to dozens of minutes. All larvae receiving ablation were freed from restraining agarose and recovered to normal swimming for $>2 \mathrm{~h}$ before later recording.

Statistics. Statistical tests were performed with programs written in MATLAB (The MathWorks). Paired permutation test was performed to examine the difference between the responses of the same fish under different experimental conditions. In paired permutation, responses of 
each fish were randomly switched between the different conditions, and the mean of the differences was calculated. We repeated such permutation for 10,000 times, and each permutation resulted in a mean difference. Among these mean differences, the ratio of differences larger than that of the real data gave the $p$ value (Moore et al., 2009). For the laser ablation experiments, the difference was between two distinct groups of fish; thus, unpaired permutation test was performed. In unpaired permutation, data from the two groups were mixed together to be randomly repartitioned to two new groups of the original sizes, and then the difference of group means was calculated. We repeated such permutation for 10,000 times, and each permutation resulted in a mean difference. Among these mean differences, the ratio of differences larger than that of the real data gave the $p$ value (Moore et al., 2009). The $p$ value $<0.05$ was regarded as statistically significant.

\section{Results}

To monitor dopamine release in intact larval zebrafish, we locally applied food extract to one nostril and recorded amperometric responses in the neuropil of the ipsilateral visual center OT using C-fiber microelectrodes (with a tip length $\sim 30 \mu \mathrm{m}$; see Materials and Methods; Fig. 1A). In amperometry mode, the C-fiber microelectrode was held at $780 \mathrm{mV}$, capable of oxidizing monoamines, including dopamine, serotonin, and norepinephrine (Wang et al., 2011). An amperometric current was readily evoked when food extract, but not external solution, was applied (paired permutation test, $p<10^{-4}, n=14$ larvae; Fig. $1 B$ ), indicating its olfactory origin. This current was dependent on oxidation because no obvious response was observed when the C-fiber microelectrode was held at $0 \mathrm{mV}$ (paired permutation test, $p<10^{-4}$, $n=18$ larvae; Fig. 1C). To further identify which monoamine was involved, specific blockers of monoamine transporters were bath-applied. We found that food extract-evoked responses were significantly enhanced when larvae were treated with the dopamine reuptake blocker nomifensine (paired permutation test, $p<10^{-4}, n=8$ larvae), but not the serotonin reuptake blocker fluoxetine ( $p=0.99, n=7$ larvae) or norepinephrine reuptake blocker desipramine ( $p=0.97, n=6$ larvae) (Fig. $1 D)$, indicating that amperometric responses reflect dopamine release.

By imaging double transgenic $\operatorname{Tg}($ dat:Gal4,UAS:GFP) zebrafish larvae, in which GFP is specifically expressed in dopaminergic neurons through the promoter of the dopamine transporter gene dat, we found that dopaminergic neurons in the pretectum intensively innervated the neuropil of OTs (Fig. 2A). Interestingly, using in vivo light sheet calcium imaging of $\operatorname{Tg}$ (dat:GCaMP6s) larvae, in which the calcium indicator GCaMP6s is specifically expressed in dopaminergic neurons, we found that dopaminergic processes within the OT neuropil exhibited robust calcium activities when food extract, but not external solution, was applied to one nostril (paired permutation test, $p=0.016, n=6$ larvae; Fig. $2 B$ ), suggesting that pretectal dopaminergic neurons are olfactorily responsive and may convey olfactory information to the OT. Moreover, after two-photon laser-based ablation of pretectal dopaminergic neurons (Fig. 2C), food extract-evoked amperometric responses in the OT were largely impaired (unpaired permutation test, $p=0.017, n=13$ larvae with ablation; Fig. $2 D$ ), indicating that pretectal dopaminergic neurons are responsible for olfactory evoked dopamine release.

\section{Discussion}

In the present study, we established electrochemical recording of dopamine release in awake zebrafish larvae using C-fiber microelectrodes. To characterize an electrochemical signal in biological systems, there are several standard criteria in the field (Phillips
A

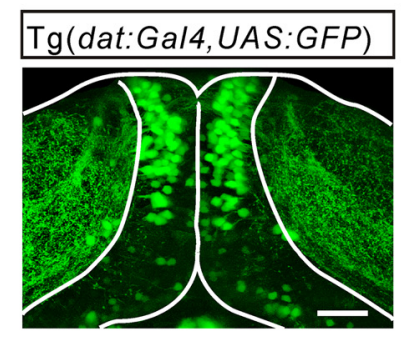

C

\section{B}

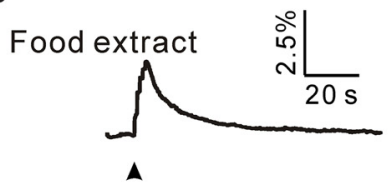

External solution

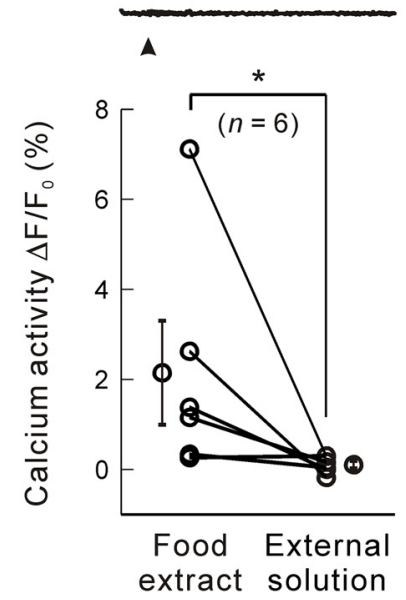

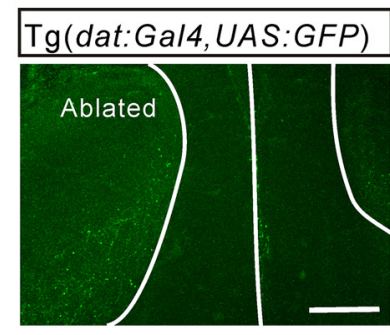

D

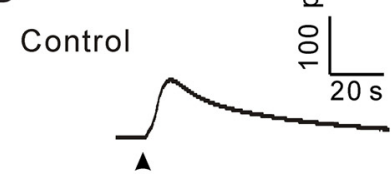

Ablated
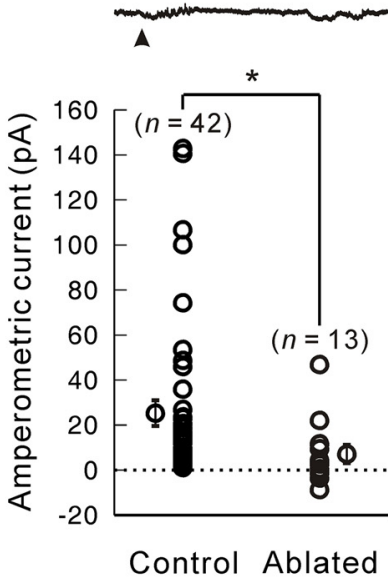

Figure 2. Pretectal dopaminergic neurons mediate food extract-evoked dopamine release at the 0T. $\boldsymbol{A}$, Projected two-photon microscopy image of a $\mathrm{Tg}($ dat:Gal4,UAS:GFP) zebrafish larva at $8 \mathrm{~d}$ post-fertilization ( $\mathrm{dpf}$ ), showing that pretectal dopaminergic neurons send processes to the neuropil of the 0T. White lines indicate the outline of the soma and neuropil layers of OTs. Up is rostral. $\boldsymbol{B}$, Food extract- or external solution-evoked calcium activities of pretectal dopaminergic neurons' processes at 0T neuropil in $\mathrm{Tg}$ (dat:GCaMP6s) larvae at $7 \mathrm{dpf}$. Top, Middle, Sample traces. Bottom, Summary. C, Projected two-photon microscopy image of an $8 \mathrm{dpf} \mathrm{Tg}$ (dat: Gal4,UAS:GFP) zebrafish larva, in which pretectal dopaminergic neurons were ablated with a two-photon laser. $\boldsymbol{D}$, Food extract-evoked amperometric current responses in larvae with intact ("Control") or ablated pretectal dopaminergic neurons ("Ablated"). Top, Middle, Sample traces. Bottom, Summary. ${ }^{*} p<0.05$. Error bars indicate SEM. Scale bars: $\boldsymbol{A}, \boldsymbol{C}, 50 \mu \mathrm{m}$.

and Wightman, 2003). The characterization of dopaminergic signal in this study well fits these criteria. First, the signal was detected when the electrode potential was held at $780 \mathrm{mV}$, but not 0 $\mathrm{mV}$, indicating a Faradic current (electrochemical criterion). The signal was enhanced by a blocker specific to the dopamine transporter, but not by a blocker of norepinephrine or serotonin transporter (pharmacological criterion). It was attenuated when the pretectal dopaminergic neurons were ablated (anatomical and physiological criterion). Furthermore, pretectal dopaminergic neurons could be activated by food extract (reproducibility criterion). These lines of evidence collectively indicate that the signal is indeed dopamine.

Being a gold standard in monitoring dopamine release, electrochemical recording has provided primary understanding about how phasic dopaminergic signaling participates in various neural functions (Phillips et al., 2003; Dommett et al., 2005; Clark et al., 2010; Wang et al., 2011; Howe et al., 2013). Although larval 
zebrafish have much fewer dopaminergic neurons, several orders lower than mammals previously targeted with C-fiber microelectrodes, our method with microelectrodes is sensitive enough to monitor dopamine release from a small population of dopaminergic neurons in an intact animal, with an amperometric current as small as several picoamperes, representing its competence in zebrafish larva studies. Because of dopamine involvement in a broad spectrum of brain functions, the upstream and downstream circuits of dopaminergic systems have recently received intense study (Tay et al., 2011; Watabe-Uchida et al., 2012). With its tiny and transparent brain, zebrafish larva provides easy access to dopaminergic nuclei and their interconnected brain areas $\mathrm{Mu}$ et al., 2012). By combining dopamine release monitoring and other assays, zebrafish larvae may serve as a working model for thoroughly dissecting the functions and underlying mechanisms of dopaminergic systems.

We found that the dopaminergic neurons in the pretectum are responsible for the dopamine release in the OT evoked by food extract. The pretectum has been found to help the OT to discriminate visual stimuli relevant to prey (Jorg-Peter, 1987). Although previous work lacks knowledge about the identity of pretectal neurons, our study suggests that pretectal dopaminergic neurons convey olfactory information to the OT and cross-modally contribute to the detection of visual preys.

\section{References}

Clark JJ, Sandberg SG, Wanat MJ, Gan JO, Horne EA, Hart AS, Akers CA, Parker JG, Willuhn I, Martinez V, Evans SB, Stella N, Phillips PE (2010) Chronic microsensors for longitudinal, subsecond dopamine detection in behaving animals. Nat Methods 7:126-129. CrossRef Medline

Dommett E, Coizet V, Blaha CD, Martindale J, Lefebvre V, Walton N, Mayhew JE, Overton PG, Redgrave P (2005) How visual stimuli activate dopaminergic neurons at short latency. Science 307:1476-1479. CrossRef Medline

Friedrich RW, Jacobson GA, Zhu P (2010) Circuit neuroscience in zebrafish. Curr Biol 20:R371-R381. CrossRef Medline

Howe MW, Tierney PL, Sandberg SG, Phillips PE, Graybiel AM (2013) Prolonged dopamine signalling in striatum signals proximity and value of distant rewards. Nature 500:575-579. CrossRef Medline

Huang HP, Wang SR, Yao W, Zhang C, Zhou Y, Chen XW, Zhang B, Xiong W, Wang LY, Zheng LH, Landry M, Hökfelt T, Xu ZQ, Zhou Z (2007) Long latency of evoked quantal transmitter release from somata of locus coeruleus neurons in rat pontine slices. Proc Natl Acad Sci U S A 104: 1401-1406. CrossRef Medline

Jorg-Peter E (1987) Neuroethology of releasing mechanisms: prey-catching in toads. Behav Brain Sci 10:337-405. CrossRef

Kang X, Xu H, Teng S, Zhang X, Deng Z, Zhou L, Zuo P, Liu B, Liu B, Wu Q, Wang L, Hu M, Dou H, Liu W, Zhu F, Li Q, Guo S, Gu J, Lei Q, Lü J, et al.
(2014) Dopamine release from transplanted neural stem cells in Parkinsonian rat striatum in vivo. Proc Natl Acad Sci U S A 111:15804-15809. CrossRef Medline

Lammel S, Ion DI, Roeper J, Malenka RC (2011) Projection-specific modulation of dopamine neuron synapses by aversive and rewarding stimuli. Neuron 70:855-862. CrossRef Medline

Marder E, O'Leary T, Shruti S (2014) Neuromodulation of circuits with variable parameters: small circuits reveal principles of state-dependent and robust neuromodulation. Annu Rev Neurosci 37:329-346. CrossRef Medline

McLean DL, Fetcho JR (2004) Ontogeny and innervation patterns of dopaminergic, noradrenergic, and serotonergic neurons in larval zebrafish. J Comp Neurol 480:38-56. CrossRef Medline

Moore DS, McCabe GP, Craig BA (2009) Introduction to the practice of statistics. New York: W. H. Freeman.

Mu Y, Li XQ, Zhang B, Du JL (2012) Visual input modulates audiomotor function via hypothalamic dopaminergic neurons through a cooperative mechanism. Neuron 75:688-699. CrossRef Medline

Phillips PE, Wightman RM (2003) Critical guidelines for validation of the selectivity of in-vivo chemical microsensors. TrAC Trend Anal Chem 22:509-514. CrossRef

Phillips PE, Stuber GD, Heien ML, Wightman RM, Carelli RM (2003) Subsecond dopamine release promotes cocaine seeking. Nature 422:614618. CrossRef Medline

Rees HR, Anderson SE, Privman E, Bau HH, Venton BJ (2015) Carbon nanopipette electrodes for dopamine detection in Drosophila. Anal Chem 87:3849-3855. CrossRef Medline

Schultz W, Dayan P, Montague PR (1997) A neural substrate of prediction and reward. Science 275:1593-1599. CrossRef Medline

Tay TL, Ronneberger O, Ryu S, Nitschke R, Driever W (2011) Comprehensive catecholaminergic projectome analysis reveals single-neuron integration of zebrafish ascending and descending dopaminergic systems. Nat Commun 2:171. CrossRef Medline

Wang SR, Yao W, Huang HP, Zhang B, Zuo PL, Sun L, Dou HQ, Li Q, Kang XJ, Xu HD, Hu MQ, Jin M, Zhang L, Mu Y, Peng JY, Zhang CX, Ding JP, Li BM, Zhou Z (2011) Role of vesicle pools in action potential patterndependent dopamine overflow in rat striatum in vivo. J Neurochem 119: 342-353. CrossRef Medline

Watabe-Uchida M, Zhu L, Ogawa SK, Vamanrao A, Uchida N (2012) Whole-brain mapping of direct Inputs to midbrain dopamine neurons. Neuron 74:858-873. CrossRef Medline

Wise RA (2004) Dopamine, learning and motivation. Nat Rev Neurosci 5:483-494. CrossRef Medline

Xu H, Zuo P, Wang S, Zhou L, Sun X, Hu M, Liu B, Wu Q, Dou H, Liu B, Zhu F, Teng S, Zhang X, Wang L, Li Q, Jin M, Kang X, Xiong W, Wang C, Zhou Z (2015) Striatal dopamine release in a schizophrenia mouse model measured by electrochemical amperometry in vivo. Analyst 140:38403845. CrossRef Medline

Zhang RW, Wei HP, Xia YM, Du JL (2010) Development of light response and GABAergic excitation-to-inhibition switch in zebrafish retinal ganglion cells. J Physiol 588:2557-2569. CrossRef Medline 